\title{
3D MHD simulations of planet migration in turbulent stratified disks
}

\author{
Ana Uribe ${ }^{1}$, Hubert Klahr ${ }^{1}$, Mario Flock ${ }^{1}$ and Thomas Henning ${ }^{1}$ \\ ${ }^{1}$ Max-Planck-Institut für Astronomie, Königstuhl 17, \\ D-69117 Heidelberg, Germany \\ email: uribe@mpia.de
}

\begin{abstract}
We performed 3D MHD numerical simulations of planet migration in stratified disks using the Godunov code PLUTO (Mignone et al. 2007). The disk is invaded by turbulence generated by the magnetorotational instability (MRI). We study the migration for planets with different mass to primary mass ratio. The migration of the low-mass planet $\left(q=M_{p} / M_{s}=10^{-5}\right)$ is dominated by random fluctuations in the torque and there is no defined direction of migration on timescales of 100 orbits. The intermediate-mass planet $\left(q=M_{p} / M_{s}=10^{-4}\right)$ can experience systematic outwards migration that was sustained for the times we were able to simulate.
\end{abstract}

Keywords. accretion disks, MHD, turbulence, planetary systems: formation

\section{Introduction}

The migration of planets in turbulent disks has been studied using MHD simulations in the cylindrical disk approximation by Nelson \& Papaloizou (2003) in the first of a series of papers and by Nelson (2005) for low mass protoplanets. Baruteau \& Lin (2010) and Laughlin et al. (2004) performed HD simulations using a stochastic forcing to model the turbulent fluctuations in the density. Contrary to laminar disk migration, low mass planets migrate stochastically and depending on the amplitude of the artificial forcing, inwards migration can be slowed down for more massive planets. We study the effect of turbulence on migration using global simulations of stratified magnetized disks.

We simulate a global stratified disk in spherical coordinates $(r, \theta, \phi)$, where the computational domain is given by $r \in[1,10], \theta \in[\pi / 2-0.3, \pi / 2+0.3]$ and $\phi \in[0,2 \pi]$, the grid resolution is $\left(N_{r}, N_{\theta}, N_{\phi}\right)=(256,128,256)$ and the grid is centered in the center of mass. The ratio of the pressure scale height $H$ to the radial coordinate of the disk is taken to be a constant such that $h=H /(r \sin \theta)=0.07$. Before introducing the planet in the simulations, a weak toroidal magnetic field is imposed on the disk with constant $\beta=25$. The planet is introduced after the saturation of the MRI. The equations of motion are integrated with a leap frog integrator. The numerical setup follows the setup presented in Flock et al. (2010).

\section{Results}

Figure 1 shows the cumulative torque for simulations with a planet where $q=10^{-4}$, for different locations in the disk. We see that in both simulations there is an initial stage where the torque is negative, followed by a reversal of the direction of the migration where the torque becomes positive and takes a defined value for the rest of the simulated time. The Hill radius of the planet and the horseshoe region are resolved (by approximately 4 and 7 grid cells per half width for $r_{p}=3.3$ and $r_{p}=5.0$ respectively). In this case, the 

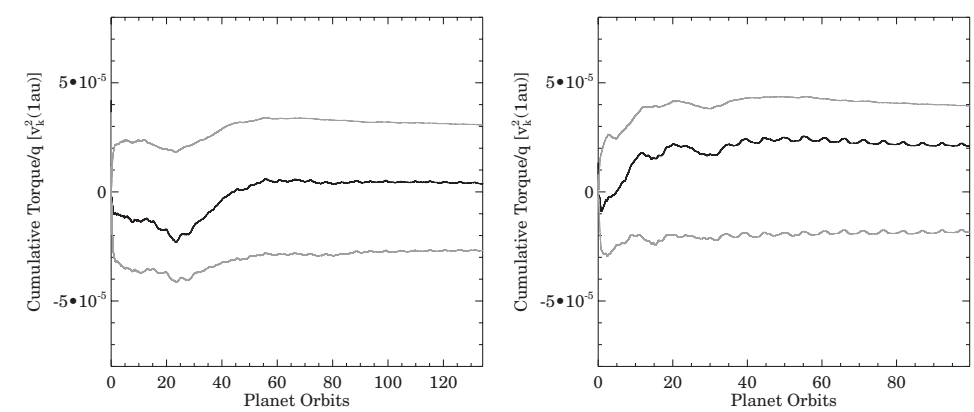

Figure 1. Cumulative torque vs planet orbits for a planet with mass ratio $q=M_{p} / M_{\text {star }}=10^{-4}$ located at $r_{p}=3.3$ (left) and at $r_{p}=5.0$ (right). A negative(positive) torque indicates inwards(outwards) migration. The gray lines shows the torque exerted by the disk inside (positive) and outside (negative) of the planet's orbit.

component of the torque originating from the horseshoe region can dominate if there is a mechanism for keeping the corotation torque unsaturated and the local density profile differs from the global profile, possibly increasing outwards, such that the corotation torque can be larger than the Lindblad torque, making the total torque positive. In our simulations the turbulence is responsible for unsaturating the corotation torque and for both simulations we see that the torque reversal ocurrs when the local surface density profile is less steep than the global $\Sigma(r) \sim r^{-0.5}$, being close to flat or increasing outwards. This is consistent with the reversal of migration that has been observed in a certain range of the paramenter space, in disks where the viscosity is modelled as an alpha viscosity (Masset et al. 2006a).

For simulations where $q=10^{-5}$, during the simulated time, migration was dominated by random fluctuations in the torque that can be orders of magnitude larger that what is expected for the value of the Lindblad or corotation torques for this planet mass. This is in agreement with simulations by Nelson (2005) of migration of low mass protoplanets in cylindrical disk models, where stratification is neglected.

\section{Discussion and Conclusions}

We demonstrate that it is possible for planets of around 30 Earth masses to undergo systematic outwards migration that is sustained up to 100 planet orbits. The question remains about the long term behavior of the torque, and whether this is only a transient behavior lasting for the first few hundred orbits (assuming the same local surface density profile), afterwards saturating and returning to laminar Type I values or fluctuating on longer timescales.

\section{References}

Baruteau, C. and Lin, D. N. C. 2010, ApJ, 709, 759

Flock, M., Dzyurkevich, N., \& Klahr, H. and Mignone, A. 2010, A\&A, 516, 26

Laughlin, G., Steinacker, A., \& Adams, F. C. 2004, ApJ, 608, 489

Masset, F. S., D'Angelo, G., \& Kley, W. 2006a, ApJ, 652, 730

Mignone, A., Bodo, G., Massaglia, S., Matsakos, T., Tesileanu, O., Zanni, C., \& Ferrari, A. 2007, ApJ, 170, 228

Nelson, R. P. 2005, A\&SA, 443, 1067

Nelson, R. P. \& Papaloizou, J. C. B. 2003, MNRAS, 339, 993 